\title{
DISSEMINATED CAT-SCRATCH DISEASE: A CASE REPORT
}

\author{
CHOWDHURY FI ${ }^{1}$, KASHEM MA ${ }^{2}$, HOSSAIN MA $^{3}$, AHSAN IM ${ }^{4}$, PANDIT A ${ }^{4}$, RIZWANA H ${ }^{5}$, ISLAM GY ${ }^{5}$
}

\begin{abstract}
:
Cat-scratch disease is an infectious disease caused by Bartonella henselae, a gram-negative bacillus. The disease is usually transmitted to the human being by cat scratches. Cat-scratch disease typically presents with painful regional lymphadenopathy. Systemic involvement is unusual. A 35 years old lady, came with the complaints of fever, abdominal pain along with nausea, anorexia $\&$ difficulties of swallowing for 6 months. She has painful cervical lymphadenopathy. Histopathology of her lymph node biopsy shows features of cat-scratch disease.
\end{abstract}

Key Words: Cat-scratch disease, zoonotic disease.

J Dhaka Med Coll. 2013; 22(2) : 229-231.

\section{Introduction:}

Cat-scratch disease is a zoonotic disease. Cats acts as a reservoir of Bartonella henselae. Cats become infected by bite of cat fleas ${ }^{1}$. Human becomes infected by scratched, bitten or licked by cats. Patients usually present with lymphadenopathy, fever 2-4 weeks after exposure. Sometimes patients show some atypical symptoms and signs. Usually the disease is self limiting in immunocompetent person $^{1,2}$.

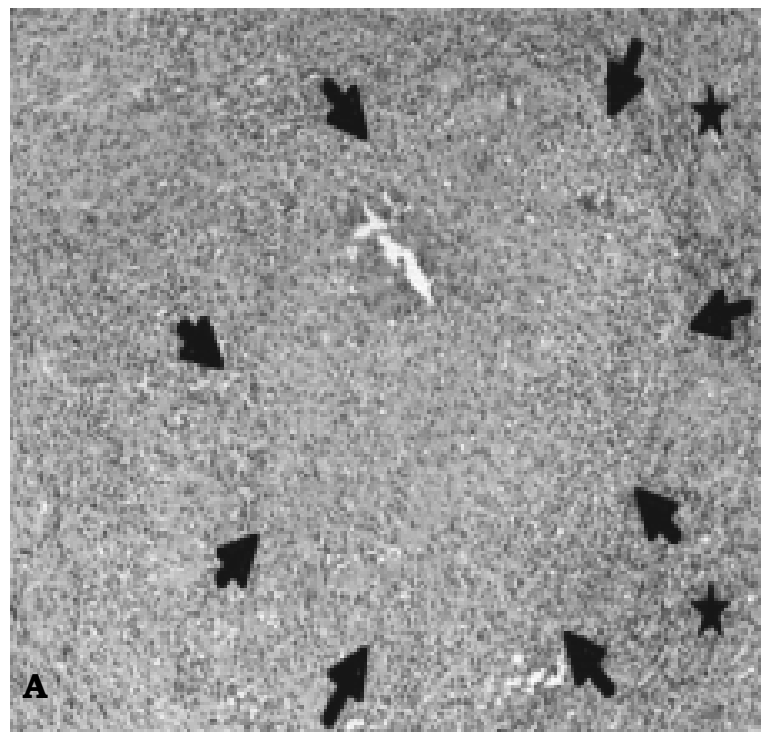

\section{Case presentation:}

A 35 years old lady, hailing from Mughda, Dhaka, came with the complaints of fever, abdominal pain along with nausea, anorexia \& vomiting, Difficulties in swallowing for 6 months. She also complains of swelling on her neck which is painful for 2 weeks. Her fever was intermittent, low grade at first but later became high grade intermittent. She was found anaemic, non ecteric, normotensive. She had bilateral cervical lymphadenopathy which was tender, discrete, soft, with no discharging

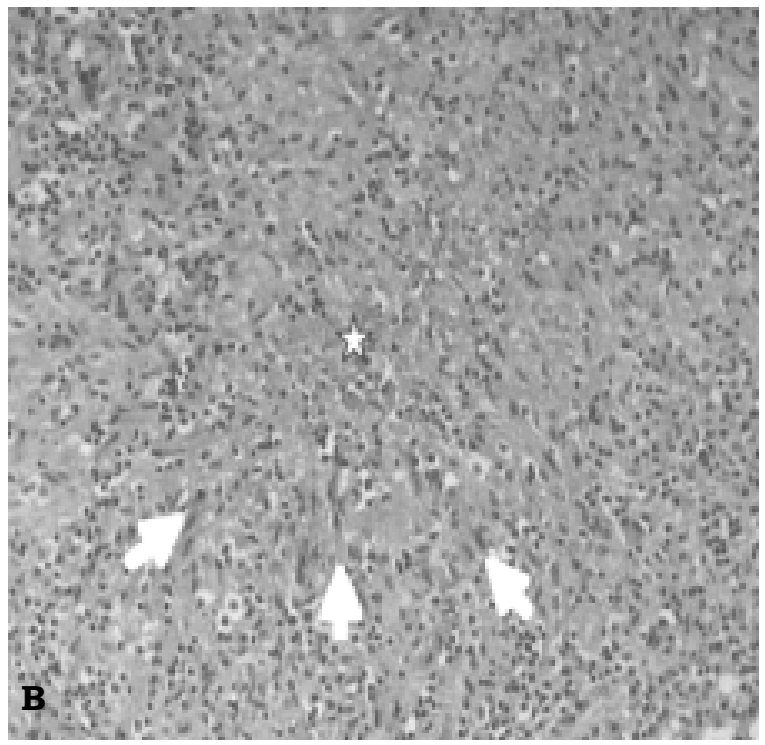

Fig.-1: The granuloma (A) appears as a well-circumscribed area (black arrows) surrounded by an inflammatory reaction with multiple lymphocytes (black asterisks); (B) Palisading epitheloid histiocytes (white arrows) surround a central stellar zone of necrosis and nuclear dust (fragmented polynuclear cells, white asterisk).

1. Prof. Faizul Islam Chowdhury, Professor of Medicine, DMCH, Dhaka

2. Dr. MA Kashem, Assistant Professor, Department of Medicine, DMCH, Dhaka

3. Dr. Md. Anwar Hossain, Assistant Professor, Department of Medicine, Sher-e-Bangla Medical College Barisal

4. Dr. Imran Munadil Ahsan, Dr. Avijit Pandit, Medial Officer, Department of Medicine, DMCH, Dhaka

5. Dr. Rizwana Halim, Dr. Gazi Yasinur Islam, Honorary Medical Officer, Department of Medicine, DMCH, Dhaka 
sinus. Her liver was just palpable but no other organomegaly. She had a scratch mark on her feet with no local lesion. Her investigation reports revealed $\mathrm{Hb}-10.7 \mathrm{~g} / \mathrm{dl}, \mathrm{WBC}-92500 /$ $\mathrm{mm}^{3}$, ESR- $101 \mathrm{~mm}$ in $1^{\text {st }}$ hour, PBF- Microcytic hypochromic anaemia, MT- Positive $(20 \mathrm{~mm})$, ANA-negative. Mild hepatomegaly, no ascites or abdominal mass or enlarged lymph nodes were detected in ultrasonography of the whole of the abdomen, and para right tracheal lymphadenopathy was found in chest x-ray. Lymph node biopsy (excision biopsy) revealed stellate abcess wth palisading of histiocytes. No malignant cell seen. The findings were consistent with cat-scratch disease. She was treated with azithromycin and responded well.

\section{Discussion:}

Cat-scratch disease was first defined as a human disease in 1931, and the term 'catscratch disease' was adopted in $1950^{1}$. It occurs throughout the world and is most common in children and young people $\mathrm{e}^{1-3}$. It is a zoonotic disease caused by genus of small Gram negative bacteria Bartonella, most commonly Bartonella henselae ${ }^{1-4}$. Cats are the reservoir hosts for Bartonella henselae. B. henselae is transmitted between cats by cat fleas (Ctenocephalides felis), probably via flea feces rather than saliva. Human becomes infected with $B$. henselae by scratches and bites from cats. More than $90 \%$ of clinical cases occur in people who have been in contact with cats, most often kittens, and the majority of these patients report having been scratched, bitten or licked ${ }^{1-3,5}$.

Cutaneous lesions usually develop at the inoculation site within 7 to 15 days after exposure, and lymphadenopathy is typically seen after 1-3 weeks. However, clinical signs have been reported as soon as 3 days and up to 50 days after exposure ${ }^{4,5}$. B. henselae appears to infect some immunocompetent people without causing clinical signs. Most others develop cat scratch disease. It may start as a small skin papule or pustule (at the site of inoculation of the organism - most commonly a cat scratch), which may rupture before healing after around three weeks. A few weeks later, usually painful local lymph node swelling develops, and in the majority of cases (85 per cent) this is limited to a single lymph node (often in the axilla or neck). The lymph node swelling may be marked, and may persist for several weeks or months ${ }^{4-6}$. In most cases no further signs develop and the swelling eventually resolves. Sometimes the affected lymph nodes form an abscess and rupture ${ }^{7}$. Other signs develop in some patients - most commonly mild fever and malaise ${ }^{1-4,6}$. Myalgia, headache and nausea are seen in some ${ }^{6,7}$. The disease in humans is typically benign and selflimiting ${ }^{1}$.

In immunocompromised individuals, the clinical signs may be more severe; complications, bacteremia and atypical presentations are more common ${ }^{7,8}$. They may develop bacillary angiomatosis and peliosis hepatis. Bacillary angiomatosis is a vascular proliferative disease of the skin and/or internal organs. Peliosis hepatis is characterized by vascular proliferation in the liver, which can result in multiple blood-filled cysts and sinusoidal dilatation ${ }^{6-8}$.

Biopsy revealed a granulomatous inflammatory disorder. Large areas of necrosis were surrounded by lymphocytes, epitheloid histiocytes and some giant cells. The epitheloid histiocytes were typically palisading around a necrotic area. This histological aspect was

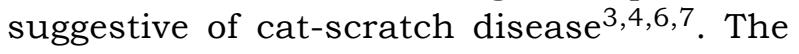
Warthin starry silver staining showed bacilli compatible with Bartonella henselae. Serological tests for $B$. henselae include various indirect immunofluorescence assays and enzyme-linked immunosorbent assays (ELISAs). A fourfold rise in titer or the presence of IgM suggests a recent infection. IgM antibodies to $B$. henselae have been reported to persist for less than three months, while IgG may be detected for more than two years ${ }^{7,8}$. Many cases of cat scratch disease in immunocompetent individuals are selflimiting. Treatment is usually supportive and symptomatic. Some other needs anitibiotic treatment. Immunocompromized are routinely treated with antibiotics ${ }^{8}$.

\section{References:}

1. Centers for Disease Control and Prevention (CDC). Available at: http://www.cdc.gov/healthypets / 
diseases/catscratch.htm [accessed on 12-092013].

2. Ratner LM, Kesack A, McCauley TR, Disler DG. Disseminated Bartonella henselae (cat-scratch disease): appearance of multifocal osteomyelitis with MR imaging. AJR 1998; 171(4): 1164-5.

3. Garcia CJ, Varela C, Abarca K, Ferrés M, Prado $\mathrm{P}$, Vial PA. Regional lymphadenopathy in catscratch disease: ultrasonographic findings. Pediatr Radiol 2000; 30(9): 640-3.

4. Fouch B, Coventry S. A case of fatal disseminated Bartonella henselae infection (cat-scratch disease) with encephalitis. Arch Pathol Lab Med 2007; 131(10): 1591-4.
5. Breitschwerdt EB. Feline bartonellosis and cat scratch disease. Vet Immunol Immunopathol 2008; 123(1-2): 167-71.

6. Zarraga M, Rosen L, Herschthal D. Bacillary angiomatosis in an immunocompetent child: a case report and review of the literature. Am J Dermatopathol 2011; 33(5): 513-5.

7. Chomel BB, Kasten RW. Bartonellosis, an increasingly recognized zoonosis. J Appl Microbiol 2010; 109(3): 743-50.

8. Breitschwerdt EB, Maggi RG, Sigmon B, Nicholson WL. Isolation of Bartonella quintana from a woman and a cat following putative bite transmission. $\mathrm{J}$ Clin Microbiol 2007; 45(1): 270-2. 